\title{
Effects of duration of type 2 diabetes mellitus on lengthening and enlargement of small abdominal aortic aneursym
}

\author{
Shijun Li, Jianguo Wang, Tingshu Yang \\ Geriatric Cardiology of Chinese PLA General Hospital, Beijing, China \\ Email: lishijun817@,126.com \\ Received 26 June 2013; revised 27 July 2013; accepted 6 August 2013 \\ Copyright (C) 2013 Shijun Li et al. This is an open access article distributed under the Creative Commons Attribution License, which \\ permits unrestricted use, distribution, and reproduction in any medium, provided the original work is properly cited.
}

\begin{abstract}
Objective: The purpose of our study aimed at evaluating the relationship of type 2 diabetes with longitudinal extension of AAA. Methods: All of 460 AAA patients aged from 41 to 92 years were retrospected in our study, and they were at least twice admitted into our hospital from January 2000 to April 2010. All patients received ultrasound measurement of aorta during each admission. Results: Our results indicated that changes of length of AAA were significantly greater in patients with diabetes than those in patients without diabetes. Type 2 diabetes was associated with length extension of AAA. In subgroup analysis, type 2 diabetes was only related to length change of small AAA rather than large AAA. Diabetes was not an independent risk factor for length extension of small AAA. There was no significant difference of glycosylated hemoglobin between diabetes patients and nondiabetes patients. Conclusions: Type 2 diabetes mellitus is closely related to length growth of small AAA, whereas diabetes is not an independent risk factor for length extension of small AAA, and serum glucose under better control could not bring about better result in limiting length extension of small AAA.
\end{abstract}

Keywords: Diabetes; Lengthening; Small Abdominal Aortic Aneurysm

\section{INTRODUCTION}

Abdominal aortic aneurysm (AAA) is defined as a permanent dilatation of the abdominal aorta and is a common and frequently lethal disease process in the elderly. The development of an AAA involved in changes in the mechanical properties of the arterial wall, which ultimately brought about widening of the vessel lumen and loss of structural integrity. Some studies argued that AAA development was associated with advanced atherosclerosis [1], and atherosclerotic risk factors such as advanced age, hypertension, smoking and hypercholesterolemia contributed to AAA progression [2-4].

Previous studies showed that the prevalence of both diabetes and AAA has risen [5-8]. Recently, a Chinese study documented that diabetes affects around $10 \%$ of the Chinese population [9]. However, after reviewing the past studies, it was showed that most of the literature regarding diabetes and AAA was aimed at the relationship between diabetes and AAA incidence. It was unclear whether diabetes was related to AAA changes in the lengthening. It was well known that length growth of AAA could possibly involve in renal artery ostia and/or downward extent to abdominal aortic bifurcation, thus increasing operative difficulty in open repair or endovascular aneurysm repair and operative expenses owing to increasing number of implanted stents and length of transplanted blood vessel. Therefore, the emphasis of our study was focused on evaluating the relationship of type 2 diabetes with longitudinal extension of AAA.

\section{MATERIALS AND METHODS}

\subsection{Study Population}

In our study, a total of 460 patients with AAA of our hospital were reviewed from the year 2000 till 2010. Their age was from 41 to 92 years, and males were 327 cases and females were 133 cases. Diameter of AAA of all patients ranged from $3.2 \mathrm{~cm}$ to $9.6 \mathrm{~cm}$. The number of patients with small AAA of less than $5 \mathrm{~cm}$ in diameter was 170 cases, and the number of patients with large AAA greater than $5 \mathrm{~cm}$ in diameter was 290 cases. 74 cases of all patients were diagnosed with diabetes. All patients received ultrasound measurement of arota during each admission and treatment with the following drugs 
or combination of some of them such as statins, angiotensin II receptor blockers, calcium channel blocker, beta-blockers or angiotensin-converting enzyme inhibittors.

\subsection{Diagnostic Criteria of Diabetes}

Diabetes mellitus was diagnosed, according to the WHO, by the classic symptoms of polyuria, polydipsia and unexplained weight loss, and/or a hyperglycaemia 11.1 $\mathrm{mmol} / \mathrm{l}(200 \mathrm{mg} / \mathrm{dl})$ in a random sample or fasting (no caloric intake for $8 \mathrm{hrs}$ ), plasma glucose $7.0 \mathrm{mmol} / \mathrm{l}$ (126 $\mathrm{mg} / \mathrm{dl})$ and/or postprandial value $11.1 \mathrm{mmol} / \mathrm{l}(200 \mathrm{mg} / \mathrm{dl})$ ( 2 hours plasma glucose level during an oral glucose tolerance test).

\subsection{Ultrasound Measurement of Abdominal Aortic Aneurysms}

Ultrasound examination of AAA was conducted by trained personnel in fast state by using color doppler ultrasound (GE Healthcare Technologies, Ultrasound, Milwaukee, WI, USA) with transducer $5 \mathrm{MHz}$. An abdominal aortic aneurysm is defined as an enlargement of the aorta of at least 1.5 times its normal diameter or greater than $3 \mathrm{~cm}$ diameter in total. Ultrasound measurement was operated by trained technicians in our hospital. Firstly, to scan transverse section of abdominal aorta, then to scan longitudinal section of abdominal aorta to observe aortic wall and lumen and shape of AAA neck, lastly to measure distances from entrance of AAA to osrium of renal artery and from exit of AAA to bifurcation of bilateral common iliac artery. Largest anteroposterior (AP) diameter was measured in longitudinal section, and largest transverse (TRV) diameter was determined in transverse section, and largest AAA length was measured from entrance of AAA to exit of AAA in longitudinal section (Figure 1). Changes of length and diameter of AP and TRV were obtained from difference

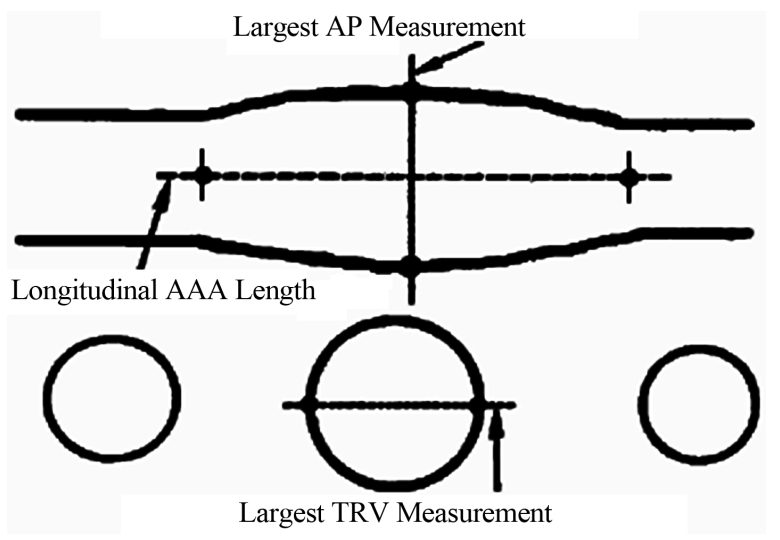

Figure 1. Ultrasound measurement of abdominal aortic aneurysms. Notes: Abdominal aortic aneurysm, AAA; Anteroposterior, AP; transverse, TRV. of measurement results determined by the last and first hospital admission.

\subsection{Other Parameters}

Height and weight were measured when the patient was admitted lastly into our hospital, and body mass index (BMI) was computed as weight divided by height squared $\left(\mathrm{kg} / \mathrm{m}^{2}\right)$. Age was recoded when patient was lastly admitted into hospital. Blood pressure was a mean value of three times measures in the last admission. The study was approved by the local ethical committee.

\subsection{Statistical Analysis}

All statistical processing was performed using SPSS-PC 17.0 (Chicago, IL, USA). All variables are presented as the mean value $\pm \mathrm{SD}$. Independent samples t-test was performed for comparative analysis of normally distributed variables, and the Mann-Whitney test was used for nonparametric analysis. Chi-square test was used to test the difference of the frequencies of associated history. Analyses of Pearson's bivariate correlation and partial correlation analysis were performed to evaluate the correlation between two parameters. Stepwise regression analysis was employed to further assess whether diabetes was an independent risk factor for length extension in AAA or not. A P value of $<0.05$ was considered as statistically significant.

\section{RESULTS}

\subsection{Clinical Characteristics of Diabetes Patients Compared with Non-Diabetes Patients}

There were significantly difference in age and ratio of gender in patients with diabetes compared those without diabetes. The prevalence of coronary artery disease, hyperlipoidemia and hypertension was obviously higher in diabetes patients than non-diabetes patients. Glycosylated hemoglobin wasn't significantly different between diabetes patients and non-diabetes patients. Changes of length of AAA were significantly increased in diabetes patients compared with those non-diabetes patients (Table 1). There wasn't of difference between patient with diabetes and those without diabetes in treatment with statins, ARB, CCB, beta-blockers or ACE inhibitors.

\subsection{Correlation Analysis between Structural Changes of AAA and Type 2 Diabetes in AAA Patients}

Bivariate correlation analysis showed that length change of AAA was closely related to history of diabetes mellitus, and wasn't associated with glycosylated hemoglobin (Table 2). Because there was significant difference between diabetes patients and non-diabetes patients in age, 
Table 1. Clinical characteristics of diabetes patients compared with non-diabetes patients $(\bar{x} \pm \mathrm{SD})$.

\begin{tabular}{|c|c|c|}
\hline & Non-diabetes patients $(\mathrm{n}=386)$ & Diabetes patients $(n=74)$ \\
\hline Age, yr & $70.89 \pm 11.63$ & $74.66 \pm 10.44^{*}$ \\
\hline Gender (Male/Female) & $338 / 48$ & $55 / 19^{*}$ \\
\hline BMI, $\mathrm{kg} / \mathrm{m}^{2}$ & $24.30 \pm 6.06$ & $24.39 \pm 2.46$ \\
\hline Observed time span, month & $17.46 \pm 25.55$ & $21.16 \pm 28.03$ \\
\hline Smoking history, \% & 20.35 & 24.07 \\
\hline History of CAD, \% & 31.05 & $56.86^{*}$ \\
\hline History of hyperlipoidemia, \% & 3.67 & $17.65^{*}$ \\
\hline History of hypertension, $\%$ & 52.57 & $66.67^{*}$ \\
\hline Systolic pressure, $\mathrm{mmHg}$ & $151.94 \pm 19.82$ & $153.40 \pm 20.54$ \\
\hline Diastolic pressure, $\mathrm{mmHg}$ & $90.14 \pm 13.02$ & $86.60 \pm 14.52$ \\
\hline \multicolumn{3}{|l|}{ Fasting serum glucose, $\mathrm{mmol} / \mathrm{L}$} \\
\hline Glycosylated hemoglobin, \% & $6.29 \pm 0.82$ & $6.17 \pm 0.99$ \\
\hline Length changes, $\mathrm{cm}$ & $1.14 \pm 1.17$ & $1.99 \pm 1.13^{*}$ \\
\hline Transverse changes, $\mathrm{cm}$ & $1.00 \pm 0.77$ & $0.86 \pm 0.41$ \\
\hline
\end{tabular}

Notes: BMI: body mass index; CAD: coronary artery disease. ${ }^{*} \mathrm{P}<0.05$ : Patients with diabetes vs Patients without diabetes.

Table 2. Bivariate correlation analysis between structural changes of AAA and type 2 diabetes in all patients with AAA.

\begin{tabular}{ccccc}
\hline & \multicolumn{3}{c}{ Bivariate correlation analysis } & Partial correlation analysis \\
\cline { 2 - 5 } & $\begin{array}{c}\text { Length } \\
\text { changes }\end{array}$ & $\begin{array}{c}\text { Transverse } \\
\text { changes }\end{array}$ & $\begin{array}{c}\text { Length } \\
\text { changes }\end{array}$ & $\begin{array}{c}\text { Transverse } \\
\text { changes }\end{array}$ \\
\hline $\begin{array}{c}\text { Diabetes } \\
\text { history, } \%\end{array}$ & $0.295^{*}$ & -0.087 & $0.343^{*}$ & 0.047 \\
\hline
\end{tabular}

Notes: Control Variables: age, gender, history of coronary artery disease, hypertension and hyperlipoidemia. ${ }^{*} \mathrm{P}<0.05$ : Correlation is significant at the 0.05 level (2-trailed).

gender, history of coronary artery disease, hyperlipoidemia and hypertension in our study. To exclude impacts of these confounding factors on length of AAA, partial correlation analysis was employed. The result confirmed that diabetes was still related to length extension of AAA (Table 2). Since biological behavior of small AAA was different from large AAA. To further elucidate relationship of diabetes with length changes of small and large abdominal aortic aneurysms, we performed correlation analysis of subgroup. The results showed that type 2 diabetes was only related to length change of small AAA rather than large AAA (Table 3).

\subsection{Clinical Characteristics of Diabetes Patients Compared with Non-Diabetes Patients in All Patients with Small Abdominal Aortic Aneurysm}

In all patients with small AAA, length and anteroposte-
Table 3. Correlation analysis of between changes of AAA and type 2 diabetes mellitus in small and large AAA patients.

\begin{tabular}{ccccc}
\hline & \multicolumn{2}{c}{$\begin{array}{c}\text { Small abdominal aortic } \\
\text { aneurysm }\end{array}$} & \multicolumn{2}{c}{$\begin{array}{c}\text { Large abdominal aortic } \\
\text { aneurysm }\end{array}$} \\
\cline { 2 - 5 } & $\begin{array}{c}\text { Length } \\
\text { change }\end{array}$ & $\begin{array}{c}\text { Transverse } \\
\text { change }\end{array}$ & $\begin{array}{c}\text { Length } \\
\text { change }\end{array}$ & $\begin{array}{c}\text { Transverse } \\
\text { change }\end{array}$ \\
\hline $\begin{array}{c}\text { Diabetes } \\
\text { history, } \%\end{array}$ & $0.606^{* *}$ & 0.156 & 0.202 & -0.161 \\
HbA1c & -0.028 & -0.696 & -0.317 & -0.272 \\
\hline
\end{tabular}

Notes: HbAlc: glycosylated hemoglobin. ${ }^{* *}$ Correlation is significant at the 0.01 level (2-tailed).

rior changes of small AAA increased in diabetes patients compared with non-diabetes patients. There wasn't significant difference in transverse changes between diabetes patients and non-diabetes patients. Glycosylated hemoglobin wasn't different in diabetes patients compared with non-diabetes patients (Table 4).

\subsection{Multiple Stepwise Regression Analysis of Length of Small AAA as Dependend Variable}

To identify whether diabetes was independent risk factor for length extension of small AAA, we perform multiple stepwise regression analysis. The results showed that only observational time span entered into multiple stepwise regression model, and diabetes wasn't independent risk factor for length extension of small AAA (Table 5). 
Table 4. Clinical characteristics of diabetes patients compared with non-diabetes patients in all patients with small abdominal aortic aneurysm. $(\bar{x} \pm \mathrm{SD})$.

\begin{tabular}{ccc}
\hline & $\begin{array}{c}\text { Non-diabetes patients } \\
(\mathrm{n}=57)\end{array}$ & $\begin{array}{c}\text { Diabetes patients } \\
(\mathrm{n}=17)\end{array}$ \\
\hline Length change & $0.58 \pm 0.71$ & $1.94 \pm 1.00^{*}$ \\
Transverse change & $0.63 \pm 0.35$ & $0.75 \pm 0.30$ \\
HbA1c & $5.80 \pm 0.46$ & $6.40 \pm 0.85$ \\
\hline
\end{tabular}

Notes: HbAlc: glycosylated hemoglobin. ${ }^{*} \mathrm{P}<0.05$ : Patients with diabetes vs Patients without diabetes.

Table 5. Stepwise regression analysis of length changes in small abdominal aortic aneurysm as dependend variable.

\begin{tabular}{|c|c|c|c|c|c|c|}
\hline & \multirow{2}{*}{ Model } & \multicolumn{2}{|c|}{$\begin{array}{l}\text { Unstandardized } \\
\text { Coefficients }\end{array}$} & \multirow{2}{*}{$\begin{array}{c}\text { Standardized } \\
\text { Coefficients }\end{array}$} & \multirow[t]{2}{*}{$\mathrm{t}$} & \multirow{2}{*}{ Sig. } \\
\hline & & B & Std. Error & & & \\
\hline \multirow{2}{*}{1} & (Constant) & 0.597 & 0.229 & & 2.605 & 0.012 \\
\hline & Time span & 0.022 & 0.005 & 0.559 & 4.527 & 0.000 \\
\hline
\end{tabular}

Notes: Dependent variable: Length changes; B: Unstandardized coefficients; Beta: Standardized coefficients.

\section{DISCUSSION}

The predominant risk factors, such as male sex, increasing age, smoking, hyperlipidaemia, hypertension and a family history, were attributed to the development of AAA $[10,11]$. Most of the literature regarding relationship of diabetes and AAA was aiming at whether diabetes was involved in the incidence of AAA. Little studies focused on association between diabetes and aortic diameter enlargement, and suggested inverse relationship between diabetes and aortic diameter in men over 65 years $[12,13]$, and treatment of hyperglycemia could limit experimental aortic aneurysm enlargement in ApoE(-/-) mice [14].

To date it was unclear whether AAA length extension was related to diabetes. Because length growth of AAA could possibly involve in renal artery ostia and/or downward extent to abdominal aortic bifurcation, thus increasing operative difficulty in open repair or endovascular aneurysm repair and operative expenses owing to increasing number of implanted stents and length of transplanted blood vessel. Therefore, it is important to investigate the relationship of type 2 diabetes with longitudinal extension of AAA.

In our study, all patients were classified as diabetes group and non-diabetes group to evaluate the role of diabetes mellitus played in longitudinal extension of AAA. The results disclosed that the length of AAA was significantly increased in diabetes patients compared with non-diabetes patients. Bivariate correlation analysis indicated that the length extension of AAA was closely related to diabetes, which indicated that diabetes was closely related to length of AAA. In our study, there was significant difference between diabetes patients and non-diabetes patients in age, gender, history of coronary artery disease, hyperlipoidemia and hypertension. To exclude impacts of these confounding factors on length of AAA, partial correlation analysis was employed. The result confirmed that diabetes was still related to length growth of AAA. Since small AAA was different from large AAA in biological behavior, small aneurysms are prone to growth and rupture. Aneurysm rupture is more likely to occur in aneurysms with larger absolute diameter growth [15]. Therefore, we performed correlation analysis of subgroup and further evaluated the relationship of diabetes with the lengthening of small AAA and large AAA respectively. The results showed that type 2 diabetes was only related to length change of small AAA rather than large AAA. In all patients with small AAA, length changes of small AAA obviously were increased in diabetes patients compared with non-diabetes patients. These results suggest that diabetes is closely related to length increase in the early development of AAA, and possibly actively controlling blood glucose could limit the lengthening of small AAA. To further identify whether diabetes was an independent risk factor for longitudinal extension of small AAA, we perform multiple stepwise regression. The result showed that type 2 diabetes was not an independent risk factor for length extension of small AAA, and multiple factors possibly predisposed to longitudinal extension of small AAA.

The hemoglobin test can reveal average blood glucose over a period of two to three months and is often used in setting and achieving treatment goals [16]. In our study, we observed that there was no significant difference of glycosylated hemoglobin between diabetes patients and non-diabetes patients, which possibly suggested that serum glucose under better control could not bring about better result in limiting AAA extension.

Color doppler ultrasound with transducer $5 \mathrm{MHz}$ was employed to examine changes of length and anteroposterior and transverse diameter of AAA by trained personnel in our study. The objectivity of ultrasound examination for AAA aroused controversy. At present, the majority of scholars agree that ultrasound is the standard imaging tool; if performed by trained personnel, it has sensitivity and specificity approaching 100 and 96 percent, respectively, for the detection of infrarenal AAA [17]. All ultrasound technicians of our hospital received systemic and formal training, therefore, ultrasound examination for AAA can provide reliable results in our study.

In conclusion, type 2 diabetes mellitus is closely related to length growth of small AAA, whereas diabetes is not an independent risk factor for length extension of 
small AAA, and serum glucose under better control could not possibly bring about better result in limiting AAA extension.

\section{ACKNOWLEDGEMENTS}

The authors gratefully acknowledge the staff of the Geriatric Cardiovascular Division of Chinese PLA General Hospital for their helpful suggestions and for collecting the clinical data. This research did not receive any specific grant from any funding agency in the public, commercial, or not-for-profit sector.

\section{REFERENCES}

[1] Golledge, J. and Norman, P.E. (2010) Atherosclerosis and abdominal aortic aneurysm. Cause, response, or common risk factors? Arteriosclerosis, Thrombosis, and Vascular Biology, 30, 1075-1077. doi:10.1161/ATVBAHA.110.206573

[2] Alcorn, H.G., Wolfson Jr., S.K., Sutton-Tyrrell, K., Kuller, L.H. and O'Leary, D. (1996) Risk factors for abdominal aortic aneurysms in older adults enrolled in the Cardiovascular Health Study. Arteriosclerosis, Thrombosis, and Vascular Biology, 16, 963-970. doi:10.1161/01.ATV.16.8.963

[3] van der Vliet, J.A. and Boll, A.P. (1997) Abdominal aortic aneurysm. Lancet, 349, 863-866. doi:10.1016/S0140-6736(96)07282-0

[4] Blanchard, J.F., Armenian, H.K. and Friesen, P.P. (2000) Risk factors for abdominal aortic aneurysm: Results of a case-control study. American Journal of Epidemiology, 151, 575-583. doi:10.1093/oxfordjournals.aje.a010245

[5] Mokdad, A.H., Ford, E.S., Bowman, B.A., Nelson, D.E., Engelgau, M.M., Vinicor, F. and Marks, J.S. (2000) Diabetes trends in the U.S.: 1990-1998. Diabetes Care, 23, 1278-1283. doi:10.1093/oxfordjournals.aje.a010245

[6] Sorensen, T.I. (2000) The changing lifestyle in the world. Body weight and what else? Diabetes Care, 23, B1-B4.

[7] Bengtsson, H., Bergqvist, D. and Sternby, N.H. (1992) Increasing prevalence of abdominal aortic aneurysms. A necropsy study. European Journal of Surgery, 158, 1923.

[8] Silverberg, E., Boring, C.C. and Squires, T.S. (1990) Cancer statistics, 1990. CA: A Cancer Journal for Clini- cians, 40, 9-26. doi:10.3322/canjclin.40.1.9

[9] Yang, W., Lu, J., Weng, J., Jia, W., Ji, L., Xiao, J., Shan, Z., Liu, J., Tian, H., Ji, Q., Zhu, D., Ge, J., Lin, L., Chen, L., Guo, X., Zhao, Z., Li, Q., Zhou, Z., Shan, G. and He, J. (2010) Prevalence of diabetes among men and women in China. The New England Journal of Medicine, 362, 1090-1101. doi:10.1056/NEJMoa0908292

[10] Shantikumar, S., Ajjan, R., Porter, K.E. and Scott, D.J. (2010) Diabetes and the abdominal aortic aneurysm. The European Journal of Vascular and Endovascular Surgery, 39, 200-207. doi:10.1016/j.ejvs.2009.10.014

[11] Lanchard, J.F. (1999) Epidemiology of abdominal aortic aneurysms. Epidemiologic Reviews, 21, 207-221.

[12] Le, M.T., Jamrozik, K., Davis, T.M. and Norman, P.E. (2007) Negative association between infra-renal aortic diameter and glycaemia: The health in men study. E The European Journal of Vascular and Endovascular Surgery, 33, 599-604. doi:10.1016/j.ejvs.2006.12.017

[13] Thompson, A., Cooper, J.A., Fabricius, M., Humphries, S.E., Ashton, H.A. and Hafez, H. (2010) An analysis of drug modulation of abdominal aortic aneurysm growth through 25 years of surveillance. Journal of Vascular Surgery, 52, 55-61. doi:10.1016/j.jvs.2010.02.012

[14] Miyama, N., Dua, M.M., Yeung, J.J., Schultz, G.M., Asagami, T., Sho, E., Sho, M. and Dalman, R.L. (2010) Hyperglycemia limits experimental aortic aneurysm progression. Journal of Vascular Surgery, 52, 975-983. doi:10.1016/j.jvs.2010.05.086

[15] Chmayssani, M., Rebeiz, J.G., Rebeiz, T.J., Batjer, H.H. and Bendok, B.R. (2011) Relationship of growth to aneurysm rupture in asymptomatic aneurysms $\leq 7 \mathrm{~mm}$ : A systematic analysis of the literature. Neurosurgery, $\mathbf{6 8}$, 1164-1171.

[16] Colman, P.G., Goodall, G.I., Garcia-Webb, P., Williams, P.F. and Dunlop, M.E. (1997) Glycohaemoglobin: A crucial measurement in modern diabetes management. Progress towards standardisation and improved precision of measurement. Medical Journal of Australia, 167, 96-98.

[17] Kent, K.C., Zwolak, R.M., Jaff, M.R., Hollenbeck, S.T., Thompson, R.W., Schermerhorn, M.L., Sicard, G.A., Riles, T.S. and Cronenwett, J.L. (2004) Screening for abdominal aortic aneurysm: A consensus statement. Journal of Vascular Surgery, 39, 267-269. doi:10.1016/j.jvs.2003.08.019 